\title{
HALLAZGOS DE UNA INVESTIGACIÓN EN COMUNICACIÓN Y DESARROLLO EN LA REGIÓN ANDINA COLOMBIANA ${ }^{1}$
}

\author{
FINDINGS OF A COMMUNICATION RESEARCH AND \\ DEVELOPMENT IN THE COLOMBIAN ANDES
}

\author{
Dorys Andrea Sotelo Carreño 2
}

\begin{abstract}
RESUMEN
El artículo da cuenta de los resultados generales y las principales conclusiones de una investigación interinstitucional en el área de la comunicación y el desarrollo, hecha por docentes de los programas de comunicación social de la Universidad Nacional Abierta y a Distancia (UNAD), la Universidad Santo Tomás (USTA) y la Universidad Minuto de Dios, cuyo objetivo en una primera fase, fue mapear dichas experiencias para identificar cuáles, cuántas y en dónde estaban; finalmente, se detectaron 250, de las cuales 112 contestaron una encuesta que diseñó el equipo investigador.
\end{abstract}

En una segunda fase fueron seleccionadas 10 de esas experiencias, a las que se les hicieron estudios de caso, con relatos de vida a los líderes. Las experiencias estaban ubicadas en Mutiscua (Norte de Santander), San Vicente de Chucurí (Santander), San Antonio del Prado, Medellín (Antioquia), Tibasosa (Boyacá), Sibaté (Cundinamarca), Montenegro (Quindío), Neiva (Huila), Guadalajara de Buga (Valle del Cauca), Pitalito (Huila) y Popayán (Cauca).

La investigación conjugó metodologías cuantitativas y cualitativas para mapear y comprender experiencias de comunicación y desarrollo relacionadas con el cuidado y optimización de los recursos naturales y las problemáticas medioambientales en general, con las implicaciones conflictivas generadas por las políticas de desarrollo, desde la visión industrial y tecnológica. Apunta a tener una clara idea de los procesos de comunicación orientados a la gestión de problemáticas medioambientales, por medio de la interacción de diversos actores sociales con sus potencialidades, en aras de objetivos comunes y de la consolidación de un sentido de lo público frente a los conflictos medioambientales.

Palabras clave: Cambio social, comunicación, desarrollo, mapeo, medio ambiente, sostenibilidad.

\section{ABSTRACT}

The article reports on the overall results and key findings of an investigation agency in the area of communication and development, done by teachers of social communication programs of the National Open University and Distance (UNAD), the Santo Tomas University and the University

\footnotetext{
1 Artículo surgido de la investigación intitulada 'Experiencias de comunicación y desarrollo sobre medio ambiente-Estudios de caso en la región Andina de Colombia', que se desarrolló gracias a un convenio celebrado entre la Universidad Nacional Abierta y a Distancia (UNAD), la Universidad Santo Tomás y la Corporación Universitaria Minuto de Dios. Los investigadores son: Patricia López Preciado, Germán Sáenz, Andrea Sotelo, Eliana Herrera, Victoria Rugeles, Carlos Andrés Muñoz, Ana María Guerrero, María Isabel Noreña y Yenny Parra.

2 Dorys Andrea Sotelo Carreño: Tutora de la Escuela de Ciencias Sociales, Artes y Humanidades de la Universidad Nacional Abierta y a Distancia. Correos electrónicos: andsot33@hotmail.com, doris.sotelo@unad.edu.co.
} 
Minuto de Dios, whose objective at an early stage was to map these experiences to identify what, how and where they are. Finally, 250 were found, of which 112 answered to a survey designed by the research team.

In a second phase, we selected 10 of those experiences, to case-studies and life stories of leaders, which are located in Mutiscua (Norte de Santander), San Vicente de Chucuri (Santander), San Antonio del Prado, Medellin (Antioquia), Tibasosa (Boyaca), Sibate (Cundinamarca), Montenegro (Quindio), Neiva (Huila), Guadalajara de Buga (Valle del Cauca), Pitalito (Huila) and Popayan (Cauca).

In sum, the research combines quantitative and qualitative methodologies to map and understand communication and development experiences related to the care and optimization of natural resources and environmental issues in general, generate conflicting implications of development policies, from the viewpoint industrial and technological. It aims at getting a clear idea of the communication processes that seek to manage environmental issues through the interaction of various social actors with their potential, for the sake of common goals and the consolidation of a sense of public versus to environmental conflicts.

Key words: Communication, development, environment, mapping, social change, sustainability.

Recibido: 4 de octubre de 2011 Aceptado: 6 de diciembre de 2011

\section{INTRODUCCIÓN}

Este artículo tiene como fundamento la investigación intitulada 'Experiencias de comunicación y desarrollo sobre medio ambiente. Región andina colombiana', la cual fue desarrollada en los años 2009 y 2010 en el marco de un convenio interinstitucional firmado entre las Universidades UNAD, Minuto de Dios y USTA, cada una de las cuales aportó tres investigadores para que indagaran acerca de la forma como se comprendía la relación comunicación y desarrollo en experiencias cotidianas adelantadas por comunidades. El interés se centró en reconocer cómo se vivencian los procesos de comunicación y desarrollo en las comunidades de base y las organizaciones sociales que lideran proyectos en diferentes territorios del país. Se focalizó la sistematización y el análisis desde el problema del medio ambiente y la sostenibilidad, por ser uno de los temas de mayor interés en el contexto nacional, debido al fenómeno del calentamiento global que registra el planeta.

Estas tres universidades en sus programas de comunicación hacen énfasis en problemáticas sociales. La USTA lo hace en la paz; la UNAD, en lo social-comunitario, y UNIMINUTO, en la participación y la ciudadanía. Ellas están interesadas en estudiar la manera como la comunicación está cumpliendo un papel de gestora e impulsora de procesos de cambio social, es decir, de mejoramiento de las condiciones de vida de las comunidades, de forma particular en lo relacionado con el medio ambiente.

\section{Una rápida panorámica a la investigación}

El trabajo de investigación buscaba responder de manera central este interrogante: ¿Cómo se entiende la relación 
comunicación-desarrollo desdeexperiencias mplementadas por comunidades que buscan la gestión y transformación social de problemáticas relacionadas con el medio ambiente en Colombia?

Para dar respuesta a esta pregunta problema, la investigación viajó por los conceptos que las experiencias manejan de comunicación, desarrolloy medio ambiente; por la forma como interrelacionan dichos conceptos; por sus actores, el contexto en el que se desarrollan, las problemáticas ambientales que les aquejan, los medios de comunicación (masivos o alternativos) que utilizan para hacer visible su trabajo, su incidencia en la esfera pública y su impacto en la región, entre otros.

\section{METODOLOGÍA}

La propuesta fue pensada desde dos procesos que implican, además del cruce de metodologías cuantitativas y cualitativas, la reflexión complementaria entre un proceso empírico analítico y otro histórico hermenéutico.

Por un lado, se propuso un proceso macrosocial para identificar (cuántas y cuáles) y describir (características generales) todas las experiencias que se lograron ubicar en una región, mediante un sondeo o encuesta. A través de redes sociales, Ministerio de Comunicaciones, Ministerio de Cultura y Ministerio del Medio Ambiente, se ubicaron no solo experiencias mediáticas, sino también artísticas y lúdicas, para contemplar otras formas comunicativas poco estudiadas, como lo muestran los resultados de la Investigación sobre el estado del arte y el rastreo de antecedentes.

El sondeo se orientó a ubicar por regiones las experiencias de comunicación y desarrollo generadas desde organizaciones comunitarias o cualquier otro tipo de comunidades de base, a saber: colegios, escuelas, universidades e iglesias que estén activas en el momento de la recolección de la información. No se partió del criterio de la legalidad sino de la legitimidad de estos procesos en las comunidades. Se realizó la recolección de datos particulares de cada experiencia para hacer una lectura y una caracterización general de las experiencias en la región, en términos cuantitativos y cualitativos.

La encuesta que se diseñó y aplicó constaba de cuatro partes: la primera, indagaba los datos generales de la experiencia como nombre, dirección, teléfono y líderes; la segunda, preguntaba las características de la experiencia como: los procesos de comunicación utilizados, su situación legal, los años que lleva funcionando y el origen de la experiencia; la tercera se refería a la visión del medio ambiente; la cuarta, averiguaba los procesos de participación y comunicación, los actores sociales, el concepto de comunicación y las acciones o estrategias concretas de comunicación que contribuían a gestionar, resolver o comprender los problemas ambientales.

Producto de la aplicación del instrumento, se obtuvo una base de datos con 112 experiencias de comunicación, desarrollo y medio ambiente de la región andina colombiana. A partir de este primer proceso se realizó un análisis microsocial de corte histórico-hermenéutico, mediante el desarrollo de estudios de caso, con el ánimo de comprender y profundizar en algunas experiencias.

El método central fue el trabajo etnográfico, con la vinculación de estudiantes de las tres facultades en las modalidades que cada institución tenía previsto, es decir, 
asistentes de investigación, estudiantes en práctica y semilleros. Se aplicaron técnicas de observación y entrevistas. Para la selección de las experiencias se establecieron tres parámetros iniciales: primero, que el proyecto tenga tres años de actividad en la comunidad, es decir, experiencias anteriores a 2006, para poder hacer evaluaciones de implicaciones 0 incidencias en la esfera pública; segundo, se tomó un caso por cada medio y uno por las experiencias diferentes de las mediáticas; y tercero, se procuró tener al menos una experiencia por cada departamento de la región. Por decisiones presupuestales y de logística se hicieron 10 estudios de caso en la región.

\section{RESULTADOS}

Inicialmente se dará cuenta de los principales resultados obtenidos por los investigadores en la etapa de mapeo y posteriormente se expondrán los hallazgos obtenidos en los estudios de caso.

\section{Principales resultados del mapeo (cuantitativo)}

Este mapeo proporcionó al equipo investigador la información necesaria para identificar algunos de los casos existentes y poder tener criterios de valoración para la selección de los estudios de caso. Aunque en un principio se pensó en tomar únicamente experiencias con origen en organizaciones comunitarias, finalmente este mapeo le permitió al equipo avizorar otros aspectos que comparten las experiencias independientemente de su origen, reconociendo que podía ser más significativo para el proceso de comprensión de la relación comunicación, desarrollo y medioambiente, si los estudios de caso incluían experiencias de origen diverso, pero estaban com- prometidas en gestionar problemáticas medioambientales desde la lógica de la comunicación para el cambio social.

Algunos de los resultados obtenidos en esta etapa se muestran a continuación:

El lugar geográfico de la experiencia, como se observa en el gráfico 1 , presenta una alta proporción en Bogotá D. C. con un $33 \%$. El restante $67 \%$ está distribuido a lo largo de la región, aunque la proporción de experiencias representa menos del $9 \%$. Cuatro de estos lugares tienen proporciones menores al 3.6\%, como son Quindío, Risaralda, Valle del Cauca y Cauca.

Origen de la experiencia: Se detectó que las organizaciones sociales, las instituciones y los medios son los gestores iniciales de estas experiencias.

Figura 1. Origen de la experiencia según agentes gestores (Cifras Absolutas)

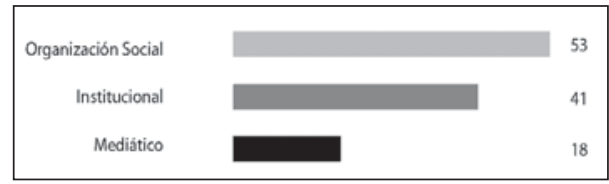

Como lo representa la figura 1 , los agentes gestores iniciales de las experiencias en comunicación de la región andina que se reconocen como el punto de origen, son organizaciones sociales en un $47.3 \%$ e institucionales en un $36.6 \% \mathrm{y}$, aunque estas son las de mayor proporción, en la región también el origen está dado desde los medios como agentes en un $16.1 \%$.

Procesos de comunicación: Es de notar que quienes diligenciaron la encuesta podían marcar más de una opción. Predominan las experiencias relacionadas con talleres, espacios participativos y educativos, pues es la opción más seleccionada debido a las 
experiencias en la región. Esto da cuenta de que los procesos educativos corresponden a la mayor agrupación de respuestas de las experiencias. Los procesos educativos son seguidos por los procesos radiales, cuya explicación de las opciones es del $87.5 \%$, y los impresos en un $75 \%$. Internet es el proceso comunicativo ubicado en cuarto lugar con un $62.5 \%$.

Figura 2. Procesos de comunicación. (Cifras Absolutas)

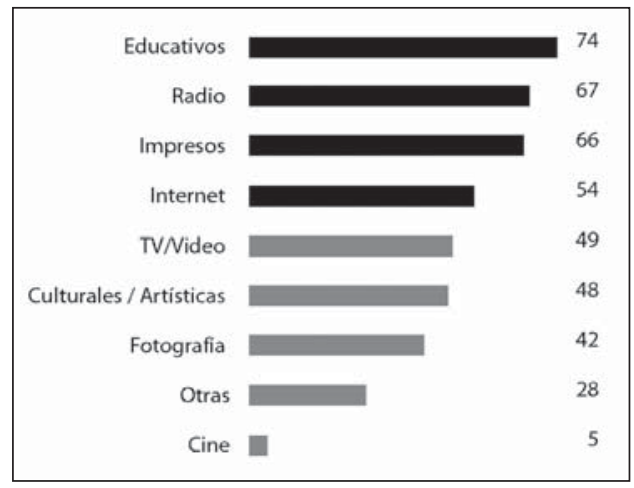

Registro legal: Como lo muestra la figura 2, el 70\% de las experiencias están registradas; sin embargo, el porcentaje de experiencias no registrado es significativo (26\%).

Tiempo de existencia: Un $20.5 \%$ tiene dos años de existencia, y un $17 \%$, tiene 10 o más años (ver figura 5). Le siguen a estos tiempos, de manera descendente, la existencia de experiencias con cinco años (11.6\%), un año y menos de un año, cada uno con un (10.7\%). Con seis años de creación se presentó un $7.1 \%$ de las experiencias. De menor proporción están las experiencias que tienen cuatro años (6.3\%), tres años (5.4\%), ocho años (3.6\%) y siete años (0.9\%). Las experiencias que no respondieron a esta pregunta corresponden a un $6.3 \%$ del total de experiencias contactadas.
Figura 3. Tiempo de la experiencia (años)

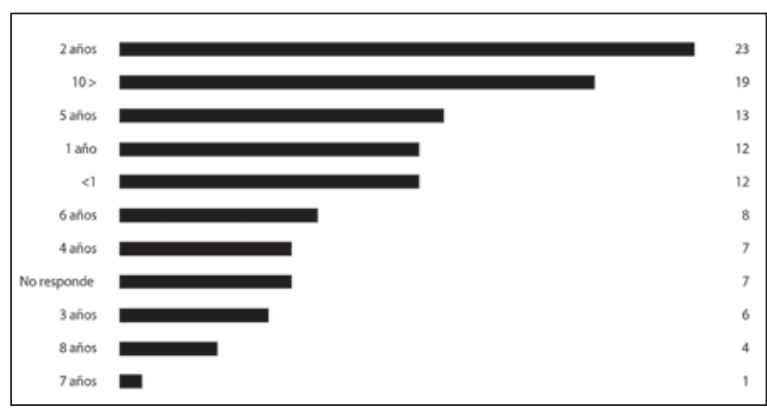

Estas experiencias presentan un promedio de cinco años de haber sido constituidas (ver figura 3), con una tendencia a que los años de las experiencias sean menores a cinco. Estos años de existencia de las experiencias presentan una desviación de 3.5 con respecto al promedio, con lo cual el coeficiente de variación es de 72, lo que indica que la heterogeneidad de los años de creación de las experiencias presenta una dispersión desconcentrada de un $72 \%$ con respecto al promedio.

En el proceso inductivo de categorización, las respuestas sobre problemáticas ambientales se organizaron entorno a tres focos de interés: 1) la protección y manejo de recursos naturales, 2 ) la concientización, sensibilización y fortalecimiento sobre problemas ambientales, y3) la problemática relacionada con el territorio.

Figura 4. Problemáticas ambientales. (Cifras Absolutas)

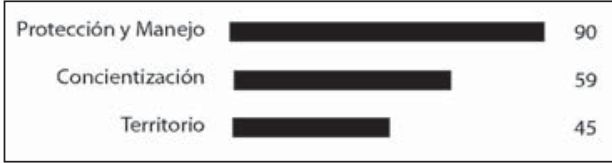

Como se observa en la figura 4, de las tres categorías obtenidas, la problemática de mayor impacto en la región es la de protección y manejo de los recursos 
naturales, seguida de la concientización de las comunidades con respecto a los problemas ambientales vividos y, finalmente, la problemática del territorio, que consiste en estrategias que propenden por recuperar el entorno de cada experiencia.

Desde la perspectiva general de los actores involucrados en las experiencias de la región, la figura 5 presenta el tipo de actor social y el número de experiencias (dentro de las 112) que los vincula en sus procesos. Pueden destacarse los actores sociales relacionados con las experiencias, provenientes de las organizaciones sociales comunitarias y de las instituciones estatales.

Figura 5. Actores sociales involucrados

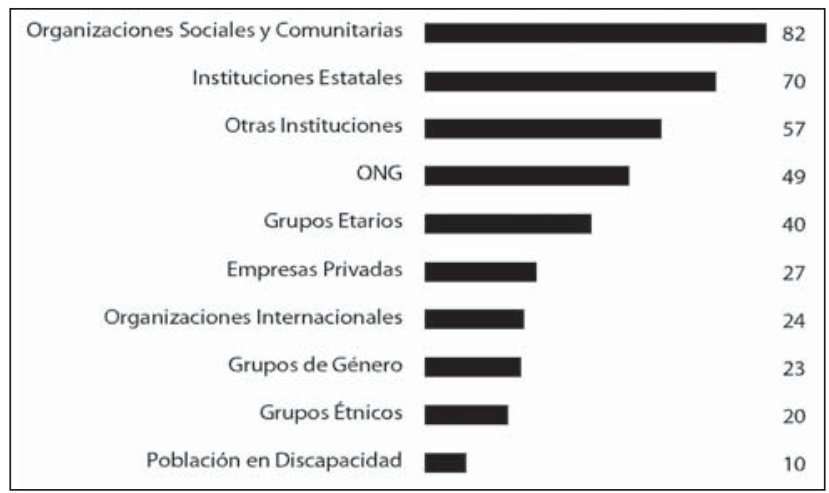

Como se representa en la figura 6 , las dificultades que han afectado las experiencias de comunicación, desarrollo y medio ambiente en la región, fueron clasificadas en cinco categorías inductivas. Dos de ellas, la responsabilidad ambiental y las dificultades económicas, son las más relevantes en cifras absolutas. Sin embargo, el análisis percentílico permite señalar que ninguna de estas problemáticas explica en mayor medida, todas las dificultades de las experiencias en la región, dada la equidad de su proporción.

Figura 6. Dificultades experimentadas por las experiencias. (Cifras Absolutas)

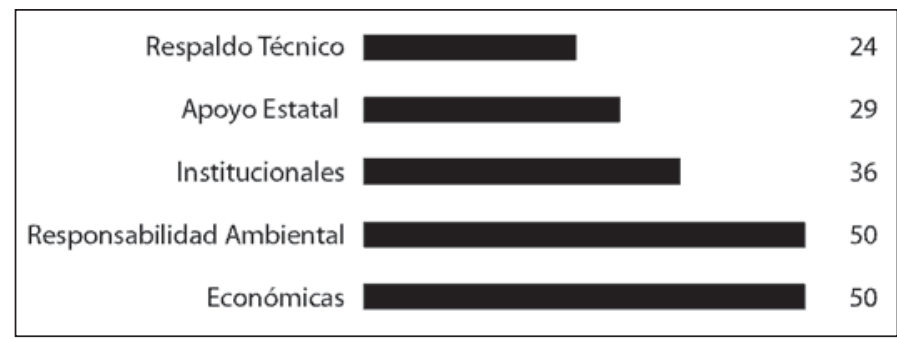

Las dificultades más relevantes en la figura son en un 75\% las económicas y de responsabilidad ambiental, mientras que las dificultades institucionales son del $50 \%$. 
Figura 7. Estrategias Comunicativas implementadas por las expericencias. (Cifras Absolutas)

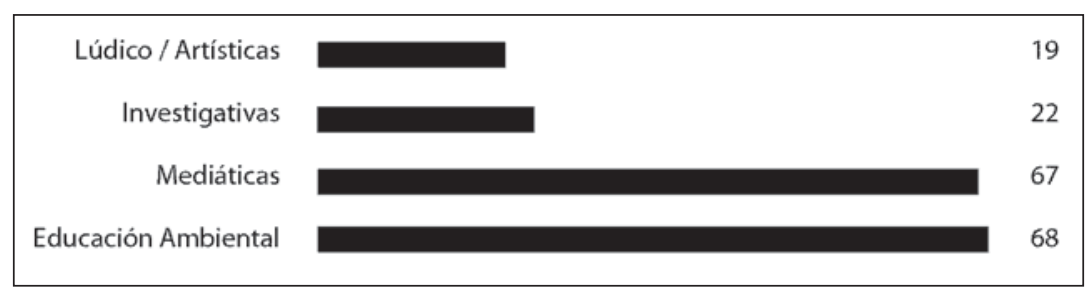

Desde la perspectiva de las estrategias comunicativas implementadas por las experiencias en comunicación en la región andina, se representa en la figura 7 la predilección por las estrategias enfocadas en la educación ambiental (68) y las estrategias mediáticas (67), mientras que las estrategias investigativas (22) y lúdico-artísticas (19) son implementadas en menor proporción.

Figura 8. Actividades participativas implementadas por las experiencias. (Cifras Absolutas)

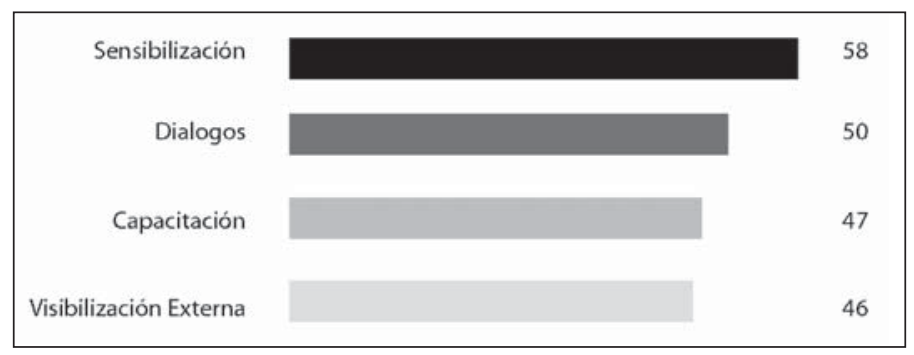

La figura 8 señala las actividades participativas que implementan las experiencias en la región. Entre estas se destacan las actividades que invitan a la sensibilización, seguidas de las relacionadas con diálogos. Las cifras que corresponden a los datos absolutos indican la cantidad de experiencias que eligieron este tipo de actividades. Dado que la respuesta era abierta y por tanto en la categorización inductiva podría resultar ser de clasificación múltiple, la suma será mayor a la totalidad de las experiencias (112).

Además, se desarrollan actividades en las que el centro de participación es la capacitación y la visibilización externa.

Figura 9. Participación de las experiencias en redes (\%)

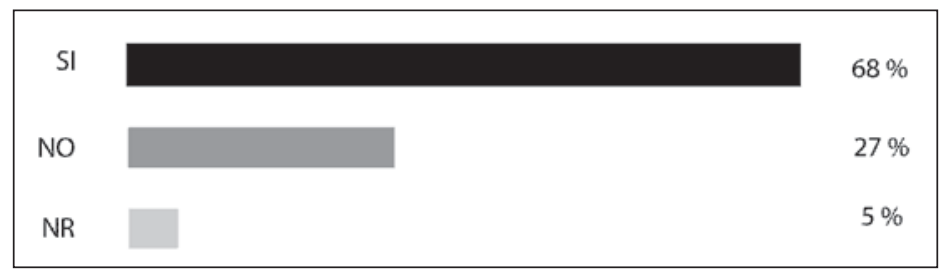


En cuanto a la participación en redes, encontramos que el $68 \%$ de las experiencias están vinculadas a alguna (ver figura 9). Solo el $27 \%$ manifiesta no hacer parte de ellas.

La figura 10 representa la mayor preferencia por participar en redes ambientales, sociales y comunitarias, mientras que se evidencia un menor interés por parte de las experiencias, en participar en redes comunicativas en la región andina (Herrera, Noreña, Guerrero, Parra, Sotelo, Sáenz, López, Rugeles y Muñoz, 2011).

\section{Principales resultados de los estudios de caso (cualitativo)}

Es preciso recordar que de las 112 experiencias que contestaron la encuesta en la etapa de mapeo, se escogieron 10 para hacerles estudios de caso. Además, se hizo un relato de vida del líder de cada proyecto. El equipo de investigación comenzó esta etapa con la selección de experiencias con más de cuatro años de labores, ubicadas en diferentes subregiones de la región andina y con una importante incidencia social, visible a través de la vinculación de diversos actores sociales, el desarrollo de diferentes actividades de participación, la preocupación por problemáticas ambientales, no solo desde la protección y manejo de recursos, sino también y prioritariamente, desde el territorio. A estos criterios se le agrega la intención de hacer visibles las experiencias que no estuvieran documentadas en investigaciones o registros de sistematización de proyectos anteriores. Finalmente, se eligieron estas experiencias:

1. Confederación Agrosolidaria Colombia, nodo central Tibasosa (Boyacá). Trabajo comunitario en red con comunidades del sector agroalimentario bajo los principios de solidaridad agroecología y comercio justo.

2. Sibaté 12.000 años de historia, Sibaté (Cundinamarca). Procesos de reconstrucción y recuperación de memoria histórica de largo aliento.

3. Emisora comunitaria Montenegro Stéreo, Montenegro (Quindío). Trabajo con fundaciones ambientales y desarrollo de campañas de concientización y sensibilización para la protección de recursos naturales.

4. Escuela de comunicación Minga del Sol, Neiva (Huila). Trabajo con grupos juveniles de comunidades vulnerables.

5. Fundación Casa de la ciencia, Guadalajara de Buga (Valle). Procesos de educación ambiental, investigación y formación científica con instituciones educativas y la comunidad. En 2009 recibió el Premio Nacional al Mérito Científico en la categoría de Divulgación de la ciencia, otorgado por la Asociación Colombiana para el Avance de la Ciencia.

6. Magazín Agenda Ambiental, Emisora La Ponderosa, Pitalito (Huila). Desarrollo de acciones educativas y participativas que evidencian la movilización de la comunidad.

7. Fundación Planeta Azul y Vida, Popayán y municipios vecinos (Cauca). Trabajo en red con radios y canales de televisión comunitaria como Balboa Estéreo, Piendamó Estéreo, Voz de Belálcazar y Ondas de Ibagué. Canales locales en Popayán, Piendamó, Santander de Quilichao y El Bordo.

8. Emisora comunitaria Nuestra Señora de las Mercedes, Mutiscua (Norte de 
Santander). Vincula en su trabajo a niños de colegios y participa propositivamente en las discusiones sobre las problemáticas medioambientales y desarrollo sostenible en el municipio.

9. Emisora Comunitaria San Vicente Stéreo, San Vicente de Chucurí (Santander). Miembro de la Asociación red de emisoras del Magdalena Medio (Aredmag).

10. Corporación Ecológica y Cultural, Ciudad Rural, San Antonio de Prado, Medellín (Antioquia). Reconoce las diversas dinámicas del territorio rural en relación permanente con lo urbano, dando protagonismo a los sujetos que lo habitan, con integración de diferentes medios.

\section{Visiones sobre comunicación}

La comunicación en estas experiencias es un entramado de ideas, situaciones, acciones, sentimientos, personas y opiniones, entre otros. Más precisamente, enlazan elementos como los líderes, sus colaboradores, las comunidades de los territoriosenlosquesedesarrollanysuvisión de mundo, las problemáticas que afectan el medio ambiente, las acciones participativas que las dinamizan para darles solución o mitigarlas, los productos comunicacionales que elaboran, los soportes tecnológicos que utilizan para divulgar sus acciones y las labores formativas y pedagógicas que movilizan las experiencias.

En ese entramado también tienen lugar factores como la acción del Estado sobre los recursos naturales, lo relacionado con el aspecto económico de la región en la que tiene asiento la experiencia y la problemática de recursos para financiar la misma. Todos estos elementos interactúan, de todos se habla, se escribe, se dialoga, se discute, se emite, se publica, se escucha, se piensa, se participa, en suma, se ponen en común con el objetivo de hacer uso de la comunicación como componente principal en la tarea de prodigar cuidado al medio ambiente en cada una de las regiones, conservar la biodiversidad, mitigar las problemáticas que afectan el medio ambiente, concientizar a las comunidades y a los gobiernos para que hagan un uso racional de los recursos naturales, educar a las comunidades sobre la necesidad de adoptar prácticas que propendan hacia la conservación del medio ambiente, posibilitar que las comunidades conozcan la riqueza de sus recursos naturales y orientar a las gentes para que cambien las prácticas agrícolas que atentan contra el medio ambiente.

La comunicación es, también para estas comunidades un dinamizador de relaciones humanas, de convivencia y de organización social y comunitaria. Además, es útil porque posibilita la transmisión de conocimiento, la formación de ciudadanos y la construcción de tejido social y contribuye a potenciar y fortalecer los procesos que están desarrollando las comunidades para mejorar su calidad de vida y resolver sus problemáticas.

En las experiencias subyace, también, un concepto de comunicación entendido como una construcción de significados y sentidos que permiten que las gentes se apropien de un territorio y se relacionen con otros y con su entorno. La comunicación se presenta como espacio de encuentro, de construcción conjunta, de diálogo activo, de toma de decisiones, de formación y de trabajo con los que hacen parte de esas organizaciones sociales como actores, emisores y receptores de ese proceso de comunicación. 


\section{Visiones sobre el desarrollo}

Es notorio en los estudios de caso que las experiencias generan una especie de robustecimiento de las comunidades con las que trabajan y a las que se proyectan; además, reflejan el fortalecimiento de la capacidad de tomar decisiones consciente y coherentemente frente a las necesidades sociales, culturales, políticas y ambientales de los pobladores, intereses reflejados en sus propuestas comunicativas, que no solo pretenden informar sino generar apropiación, como proceso pedagógico.

Los estudios de caso propician el empoderamiento a partir de sus organizaciones y exhortan a los jóvenes y niños a quienes dirigen sus propuestas, para que se concienticen de lo relevante que es mantener viva la memoria histórica de sus comunidades, afianzar la identidad con base en la apropiación del territorio y tener claro el contexto en el que actúan, para que participen en la generación de propuestas nuevas, enmarcadas dentro de las acciones que cada experiencia proyecta.

EnlaCorporaciónEcológicay Cultural Ciudad Rural y en la Escuela de Comunicación Minga del Sol, se percibe una orientación hacia el desarrollo sostenible, compartida con sus semilleros o grupos de comunicación que coordinan los líderes más jóvenes. Dicha perspectiva se materializa en recorridos temáticos, o propuestas que despiertan la curiosidad por su historia, por conocer de sus abuelos cómo era el territorio, qué sembraban, qué animales había, cómo gastaban el tiempo libre, etc.

El Museo Casa de la Ciencia en Guadalajara de Buga, por su parte, convoca a los más pequeños a aprender y hacer experimentos con los cuales comprenden temas como el cambio climático y a identificar las especies en vía de extinción y determinar posibles soluciones a estas problemáticas, lográndose así una subjetividad más sensible y consciente (Herrera et al, 2011).

El mencionado empoderamiento propicia procesos de autodeterminación tanto del individuo como de la comunidad y hace énfasis en las fortalezas de las personas, promoviendo una nueva imagen de ellas como ciudadanos con derechos y opciones. (Zambrano, Bustamante, y García, 2009). Los elementos anotados se basan en la información y el conocimiento para tomar decisiones autónomas, aspecto predominante en las experiencias estudiadas.

Una buena parte de las experiencias tiene inicio en el interés del líder que se hace manifiesto por medio de acciones que buscan dar origen a un proceso que esté orientado a dar respuesta a problemáticas de índole ambiental que se registran en el territorio en donde vive o trabaja. Así mismo, la comunicación juega en todas las experiencias un papel fundamental, pues es motor que moviliza la convocatoria, el cambio, la concientización y la reflexión; es preciso destacar que no solo se utilizan los medios masivos de comunicación, sino la comunicación alternativa y de la pedagogía (talleres, charlas, caminatas ambientales).

Se hace énfasis en las relaciones interpersonales y en la necesidad de trabajar en equipo, estar con el otro y aprender del otro, elementos que aportan nuevas significaciones en las relaciones de poder, al reconocer que el manejo de relaciones desiguales implica diferentes niveles de control sobre las propias decisiones, que generalmente son manejadas por intermediarios. En esta perspectiva, el 
grupo y su organización aparece como un elemento que dentro de un espacio social determinado, permite redensificar la vida social mediante el fortalecimiento del sujeto político, promoviendo estructuras de relacionesquehacen posiblelaparticipación democrática (La Palma y Montero, citados en Zambrano, Bustamante, y García, 2009).

En las experiencias también se evidencia la transformación del individuo en un sujeto político, a través de la participación en espacios como las mesas ambientales, los planes de desarrollo y los presupuestos participativos, así como en nuevas formas de movilización o expresión de sentires y demandas hacia los entes de poder.

El empoderamiento, entonces, se comprende como un movimiento (proceso) general de adquisición de poder con miras a alcanzar un objetivo preciso (Le Bossé y Dufort, citado en Zambrano y otros, 2009). En el ámbito comunitario se plantean dos dimensiones que lo constituyen: la autodeterminación personal, entendida como la capacidad de determinar la propia vida, y la posibilidad de participación democrática, basada en la determinación social (Herrera y otros, 2011).

Al revisar los conceptos de desarrollo identificados por las 10 experiencias, se nota que no necesariamente se deben inscribir en un modelo o paradigma, sino que refleja el accionar de las organizaciones que buscan adaptarse a los cambios del entorno.

\section{El medio ambiente}

Se aprecia en las experiencias una visión 'no antropocéntrica' del medio ambiente. No consideran al hombre el centro de la naturaleza; además, reconocen que los procesos de transformación deben estar mediados por los deberes y derechos frente a la conservación del medioambiente y el sentido de pertenencia al territorio.

Los 10 estudios de caso muestran una preocupación por recuperar y conservar la diversidad de sus recursos naturales y están orientadas a propiciar el acercamiento y la sensibilización de la comunidad a través de su participación. Lo anterior deja ver un fortalecimiento de la identidad cultural de las comunidades, a partir de la diversidad ambiental, que enmarca las costumbres, los valores y la forma como cada grupo social concibe e interpreta su mundo.

Es de destacar en las experienciasla ejecución de acciones colectivas con incidencia en la población, para masificar procesos de sensibilización y participación mediante las prácticas educativas que promueven un acercamiento a la realidad local y al mismo tiempo inciden en la conservación y preservación de la naturaleza.

Las prácticas son acciones solidarias visibilizadas en la limpieza de las calles y las riberas de los ríos, la conservación de cuencas y microcuencas, las caminatas en senderos ecológicos, el ecoturismo, el apoyo a los Proyectos Ambientales Escolares, PRAE, el fomento del conocimiento científico, la recuperación de la memoria histórica, las capacitaciones y el fomento de las prácticas agroecológicas entre prosumidores, productores y consumidores de productos orgánicos, para apoyar la sensibilización y fortalecimiento de los valores frente a lo propio, el territorio, la cultura, las problemáticas locales y regionales, la cosmovisión y el estímulo a la construcción de redes (Herrera et al, 2011).

En suma, las experiencias a las que se les hizo estudio de caso propenden por la protección, reparación y conservación 
del medio ambiente y por la mitigación de acciones como la expansión del territorio, la deforestación, el sobrepastoreo, la contaminación hídrica, el mal manejo de los residuos sólidos, el inadecuado manejo de los espacios públicos, el desconocimiento de especies endémicas y el 'ecoturismo' irresponsable. Estas experiencias muestran que las acciones comunicativas son el catalizador preciso para propiciar procesos de cambio que redunden en la protección y conservación del medio ambiente, así como en el uso racional de los recursos naturales.

\section{Relación comunicación - desarrollo - medio ambiente}

La educación ambiental es transversal a todas las experiencias estudiadas y es viabilizada a través de talleres y espacios participativos con tendencia pedagógica. Dichos procesos formativos se basan en acciones comunicativas y son considerados como cimientos sólidos del cambio de la visión de desarrollo y sus repercusiones en lo ambiental.

En los 10 estudios de caso se ve la comprensión de los procesos de comunicación-desarrollo como respuesta a problemáticas medioambientales, por medio de la vinculación de diferentes actores sociales e instituciones en los proyectos y de la búsqueda de trabajo en red. Estas prácticas participativas ligadas a la defensa del interés público sobre la preservación y cuidado del medioambiente, prioritario en la actualidad, están estrechamente ligadas con la posibilidad de generación de capital sinérgico, la capacidad social o mejor, la capacidad societal (como expresión más totalizante) de promover acciones en conjunto dirigidas a fines colectiva y democráticamente aceptados, con el conocido resultado de obtenerse así un producto final que es mayor que la suma de los componentes (Herrera et al, 2011).
Para las experiencias estudiadas, la educación ambiental es la estrategia para formar al ciudadano y romper con su desinterés por lo público, en el caso particular de esta investigación, el desinterés por una conciencia ambiental que representa un interés público. Además, las experiencias a las que se les hizo estudio de caso, asumen el tema del desarrollo como un fenómeno que genera cambios en la utilización del territorio y de los recursos naturales, los cuales a la postre, terminan por deteriorar la riqueza medioambiental de su región.

Ver a través de los lentes de las personas vinculadas a las experiencias abordadas en este estudio, es observar que existe un abanico multicolor de posibilidades de agenciamiento, empoderamiento y acción de las comunidades, orientadas a procurarse mejores condiciones de vida por medio del cuidado, conservación y uso racional de los recursos naturales, todo ello, dinamizado por la comunicación que se viste con diversos trajes, para hacer su mejor papel.

\section{DISCUSIÓN}

Al poner en diálogo los resultados de la investigación con los autores que han servido de faro guía al trabajo de indagación, es preciso traer a colación a Amparo Cadavid Bringe (2006), quien en el Congreso mundial de comunicación para el desarrollo ¿Qué comunicación para cuál desarrollo?, explica que en cualquier forma de entender el desarrollo, siempre la comunicación ha estado presente. Esta afirmación se hace palpable en las experiencias objeto de estudio, dado que sus acciones de empoderamiento de las comunidades y de generación de mejores condiciones de vida están mediadas por la comunicación. 
Las experiencias también comparten la declaración del congreso de Roma: Sin comunicación no puede existir desarrollo. Ningún intento de cambio social, de desarrollo, de participación comunitaria, de movilización social, puede soslayar la comunicación como espacio multidimensional que moviliza la conciencia y motiva la acción. En conclusión, la comunicación dota de sentido a la práctica social. En este sentido, mencionamos a Luís Ramiro Beltrán, quien resalta la importancia que tiene la comunicación en los proyectos de transformación social.

También son conocidos los trabajos de Rosa María Alfaro (1993), que entienden la comunicación y el desarrollo como un proceso que más emerge de la cultura, y por lo tanto, que hacen un cuestionamiento a fondo del tradicional concepto de desarrollo. En las experiencias abordadas en la investigación, precisamente, la relación comunicación-desarrollo y las acciones que se adelantan en este marco, surgen desde las mismas comunidades, no son impuestas por el estado ni por entes supranacionales.

También se destacan los aportes hechos al concepto de comunicación y desarrollo desde Jesús Martín Barbero (2003), quien hacia los años 80 plantea que el accionar de los medios de comunicación está mediado por la cultura de la cual hacen parte y sobre la que actúan. Estas reflexiones llevaron a los comunicadores a repensar su quehacer, pues hasta el momento quienes hacían la comunicación, estaban simplemente al servicio de quienes manejaban el desarrollo, concebido este como progreso o crecimiento económico.

Como se pudo ver en las 10 experiencias objeto de la investigación, las reflexiones en torno a la comunicación y el desarrollo se dan a partir de la cultura que las enmarca y las moviliza para generar procesos útiles que protejan el medio ambiente y contribuyan al uso racional de los recursos naturales. Cadavid (2007) señala que hay que partir de la observación de las carencias y las posiblidades, al igual que de la cultura y los imaginarios.

La mirada de las experiencias analizadas está muy deacuerdo con los planteamientos de Alfonso Gumucio Dragon (2004), en el sentido de que la comunicación se observa como dinamizadora de la construcción de redes sociales, no solo para promover el acceso al poder sino para establecer relaciones proactivas. La comunicación tiene en cuenta la acción individual de los sujetos y la capacidad de las comunidades para establecer relaciones, articular esfuerzos y recursos y potenciar sus condiciones de vida.

Desde esta perspectiva, las 10 experiencias proponen una comunicación que genera y fortalece transformaciones sociales y culturales, con el objetivo de propiciar mejores condiciones de vida de las comunidades, a partir de la interacción entre dos o más individuos o grupos y la existencia de una voluntad concertada entre quienes participan de una potencial transformación para hacerla desde el campo de la comunicación.

\section{CONCLUSIONES}

Lamayorparte(33\%)delas 112 experiencias identificadas en la etapa de mapeo están ubicadas en la ciudad de Bogotá D. C., hecho que amerita el desarrollo de una investigación particular en la capital del país, que mapee y caracterice dichas experiencia.

Son las organizaciones sociales las que principalmente le están dando origen a 
experiencias o proyectos de comunicación y desarrollo sobre medio ambiente en la zona andina colombiana; le siguen las instituciones y finalmente, los medios de comunicación.

La mayor dificultad que han encontrado quienes adelantan las experiencias o los proyectos de comunicación y desarrollo sobre medio ambiente, tiene que ver con el factor económico. De las 112 experiencias que diligenciaron el instrumento de mapeo, un $75 \%$ señalaron este ítem como su dificultad, seguido por los problemas de índole institucional.

Predominan en las 112 experiencias del mapeo, las acciones comunicativas relacionadas con talleres y espacios participativos y educativos, pues es la opción más seleccionada debido a las experiencias en la región. A los procesos educativos le siguen los procesos radiales, y a estos, los impresos.

Desde la perspectiva de las estrategias comunicativas implementadas por las 112 experiencias en comunicación en la región andina, existe una predilección por las estrategias enfocadas en la educación ambiental y las estrategias mediáticas, mientras que las estrategias investigativas y lúdico-artísticas son implementadas en menor proporción.

Al revisar los conceptos de desarrollo identificados por las 10 experiencias a las que se les hizo estudio de caso, se nota que no necesariamente se deben inscribir en un modelo o paradigma, sino que reflejan el accionar de las organizaciones que buscan adaptarse a los cambios del entorno.

La comunicación es para estas comunidades un dinamizador de relaciones humanas, deconvivenciay deorganización socialy comunitaria; además, esútil porque posibilita la transmisión de conocimiento, la formación de ciudadanos y la construcción de tejido social y contribuye a potenciar y fortalecer los procesos que están desarrollando las comunidades para mejorar su calidad de vida y resolver sus problemáticas.

La generación de conciencia ambiental y sentido de pertenencia a un territorio parten, según lo visto en las 10 experiencias, de la generación de procesos de reconciliación del ser humano con la naturaleza, de la educación, de la construcción y reconstrucción de conocimiento y de evadir toda tendencia antro-pocéntrica. La construcción de una nueva cultura del desarrollo requiere explorar nuevas formas de conocimiento conducentes al bienestar, en condiciones de equidad y armonía con el ambiente.

Las experiencias acuden a estrategias de comunicación alternativas y pedagógicas pues, si bien en algunos casos hacen uso de los mismo soportes tecnológicos de los medios masivos de comunicación, también es cierto que los colman de mensajes y contenidos diferentes, particulares $y$ originales que finalmente terminan mediando para que las comunidades en las que las experiencias de la investigación tienen injerencia, dinamicen la transformación de las maneras de ser, pensar y sentir con respecto al mejoramiento de sus condiciones de vida y del espacio vital que los contiene. En este espacio donde generaciones anteriores hicieron su plan de vida, las de hoy trazan el suyo e intentan generar las condiciones necesarias para que las generaciones venideras también puedan desarrollar su propio plan. Esta es quizá la finalidad principal que persiguen las experiencias de comunicación y desarrollo en la región 
andina de Colombia, puesto que más allá de la preocupación por el desarrollo económico, se piensa en el progreso social de las comunidades.

Las 10 experiencias permiten observar una estrecha vinculación comunicacióndesarrollo, que genera escenarios de negociación y diálogo, en los que continuamente se construyen consensos y disensos, relacionados con asuntos que les atañen a todos sus integrantes, especialmente el relacionado con el medio ambiente.

\section{REFERENCIAS}

Alfaro, R. M. (1993). Una comunicación para otro desarrollo: Para el diálogo entre el norte y el sur. Lima, Perú: Calandria.

Barbero, J. (2003). De los medios a las mediaciones. Bogotá, Colombia: Convenio Andrés Bello.

Cadavid, A. (2006). Congreso mundial de comunicación para el desarrollo. ¿Qué comunicación para cuál desarrollo?, llevado a cabo por el Banco Mundial, la FAO y The Communication Initiative en Roma, Italia.

Gumucio, A. (2004). El cuarto mosquetero: La comunicación para el cambio social. Investigación y Desarrollo, 12, (1), 2-23.

Herrera, E., Noreña, M., Guerrero, A., Parra, Y., Sotelo, A., Sáenz, G., López, P., Rugeles, M., Muñoz, C. (2011). Experiencias de Comunicación y Desarrollo Sobre Medio Ambiente: Estudios deCasoeHistorias deVida en la Región Andina de Colombia. Bogotá, Colombia: Alianza interinstitucional.

Zambrano, A., Bustamante, G. y García, M. (2009). Trayectorias organizacionales y empoderamiento comunitario: Un análisis de interfaz en dos localidades de la región de la Araucanía. Psykhe, 18, (2), 65-78. Recuperado de http://www.scielo.cl/pdf/ psykhe/v18n2/art05.pdf. 